\title{
Resonant Phase Shift from Finite Volume Interactions
}

\author{
A. C. -P. $\mathrm{Hsu}^{1}$ \\ Special Research Centre for the Subatomic Structure of Matter, The University of Adelaide \\ CSSM, Rm. 126, Lvl 1 Physics Building, University of Adelaide, SA 5005, Australia \\ E-mail: chipin.hsu@student.adelaide.edu.au
}

J. M. M. Hall

Special Research Centre for the Subatomic Structure of Matter, The University of Adelaide CSSM, Rm. 126, Lvl 1 Physics Building, University of Adelaide, SA 5005, Australia

\section{R. D. Young}

Special Research Centre for the Subatomic Structure of Matter, The University of Adelaide CSSM, Rm. 126, Lvl 1 Physics Building, University of Adelaide, SA 5005, Australia

\section{A. W. Thomas}

Special Research Centre for the Subatomic Structure of Matter, The University of Adelaide CSSM, Rm. 126, Lvl 1 Physics Building, University of Adelaide, SA 5005, Australia

A scattering process can be modelled by a finite volume lattice with appropriate boundary conditions. Such lattices have intermediate states with discretized energy spectrum. With Lüscher's formula we can obtain the phase shifts of these states. We constructed a three dimensional, non-relativistic model for the scattering of two scalar mesons on a lattice. The resonant energy spectra at different lattice sizes were obtained. Using Lüscher's formula, the corresponded phase shifts were calculated. Then, we compared the infinite volume phase shifts with the finite volume phase shifts acquired by Lüscher's formula.

The 30 International Symposium on Lattice Field Theory

Cairns, Australia

June 24-29, 2012

$1 \quad$ Speaker 
A. C. -P. Hsu

\section{Introduction}

A scattering process can be studied on a lattice with imposed periodic boundary conditions. Because of the boundary conditions, only certain discrete energy values are allowed. Hence, in a finite volume scattering process, there is a discretized energy spectrum for the intermediate states. The phase shifts of these intermediate states can be calculated by Lüscher's formula.

We construct a 3-D non relativistic model for a two meson scattering process. The energy spectrum is then calculated on the lattice with periodic boundary conditions and by using Lüscher's formula the corresponding resonant phase shifts are calculated.

Lüscher's formula can relate the phase shifts of infinite volume scattering in the real world to the energy levels observed on the finite volume. By evaluating the r-matrix we can calculate the infinite volume phase shifts for the system. In this way, we can compare the phase shifts given by Lüscher’s formula with the exact infinite volume phase shifts.

\section{Method}

We constructed a 3-D non-relativistic model for a two scalar meson scattering process with $\sigma$ as the intermediate state in a lattice with periodic boundary conditions.

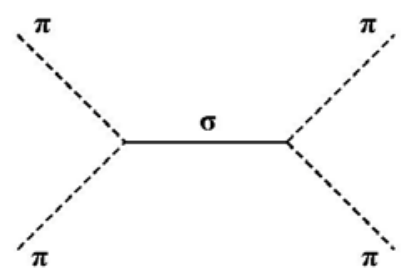

Fig. 1.

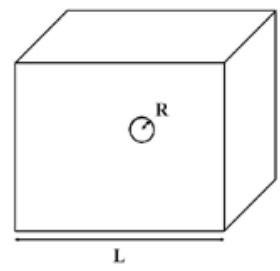

Fig. 2.

Then the momentum of the intermediate states will be discretized and is in the form

$$
k=\frac{2 \pi}{L}|\vec{n}|, \vec{n} \in \mathbf{Z}^{3}
$$

We assume that the interaction Lagrangian is

$$
L_{\text {int }}=g \sigma \pi^{2}
$$

In this interaction Lagrangian, there is no derivative term, and we can construct the Hamiltonian as

$$
\begin{gathered}
H=H_{0}+H_{I} \\
H_{I}=-L_{I}
\end{gathered}
$$

The self energy term is

$$
\Sigma_{\sigma \pi \pi}(E)=\lim _{\eta \rightarrow 0^{+}} 2 \mu \frac{\int_{0}^{\infty} d k^{\prime} k^{\prime 2} g^{2}\left(k^{\prime}\right) u^{2}\left(k^{\prime}\right)}{k^{2}-k^{\prime 2}+i \eta}
$$

where 


$$
E=2 m_{\pi}+\frac{k^{2}}{2 \mu}
$$

and $\mu$ the reduced mass of the two scalar mesons with

$$
\mu=\frac{m_{\pi}}{2}
$$

We use a Gaussian form factor for $u\left(k^{\prime}\right)$ as

$$
u\left(k^{\prime}\right)=e^{-\frac{k^{\prime 2}}{\Lambda^{2}}}
$$

With spherical symmetry, we have

$$
\int_{0}^{\infty} d k^{\prime} k^{\prime 2} f\left(k^{\prime}\right)=\frac{1}{4 \pi} \int d \Omega \int_{0}^{\infty} d k^{\prime} k^{\prime 2} f\left(k^{\prime}\right)=\frac{1}{4 \pi} \int d^{3} k^{\prime} f\left(k^{\prime}\right)
$$

By the discretization

$$
\int d^{3} k^{\prime} f\left(k^{\prime}\right) \rightarrow \sum_{n}\left(\frac{2 \pi}{L}\right)^{3} C_{n} f\left(k_{n}\right)
$$

we have

$$
\Sigma_{\sigma \pi \pi} \rightarrow 2 \mu \frac{1}{4 \pi}\left(\frac{2 \pi}{L}\right)^{3} \sum_{n} \frac{C_{n} g^{2}\left(k_{n}\right) u^{2}\left(k_{n}\right)}{k^{2}-k_{n}{ }^{2}}
$$

where $C_{n}$ is the number of dictinct 3 -D vectors $\left(n_{1}, n_{2}, n_{3}\right)$ with

$$
n_{1}^{2}+n_{2}^{2}+n_{3}^{2}=n^{2} \text { where } n_{1}, n_{2}, n_{3} \in \mathbf{Z}
$$

Take

$$
g\left(k_{n}\right)=\sqrt{\frac{1}{4 \pi}} \sqrt{\left(\frac{2 \pi}{L}\right)^{3}} \sqrt{C_{n}} g(0) e^{-\frac{\left(\frac{2 \pi}{L}\right)^{2} q^{2}}{\Lambda^{2}}}
$$

we can construct the full Hamiltonian of the scattering in the form

$$
H=\left(\begin{array}{cccc}
m_{\sigma 0} & g\left(k_{0}\right) & g\left(k_{1}\right) & \ldots \\
g\left(k_{0}\right) & 2 m_{\pi} & & \\
g\left(k_{1}\right) & & 2 m_{\pi}+\frac{k_{1}^{2}}{2 \mu} & \\
\vdots & & & \ddots
\end{array}\right)
$$

Then we can get the energy eigenvalues of the Hamiltonian. When there is no interaction, the Hamiltonian would just be

$$
H_{0}=\left(\begin{array}{cccc}
m_{\sigma 0} & 0 & 0 & \ldots \\
0 & 2 m_{\pi} & & \\
0 & & 2 m_{\pi}+\frac{k_{1}^{2}}{2 \mu} & \\
\vdots & & & \ddots
\end{array}\right)
$$


and energy eigenvalues of the non-interacting Hamiltonian are just $m_{\sigma 0}, 2 m_{\pi}, 2 m_{\pi}+\frac{k_{1}^{2}}{2 \mu} \ldots$

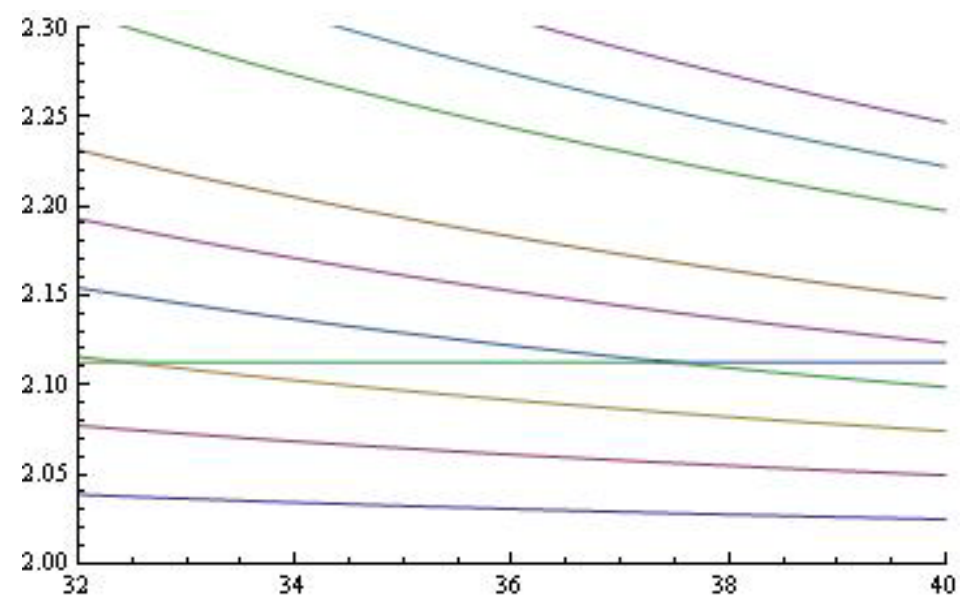

Fig. 3. Energy spectrum with no interaction and no avoided level crossing. x-axis: $L\left(1 / m_{\pi}\right)$. y-axis: resonant energy $\left(m_{\pi}\right)$.

When the interaction is turned on, the eigenvalues of the Hamiltonian exhibit avoided level crossing because of the interaction.

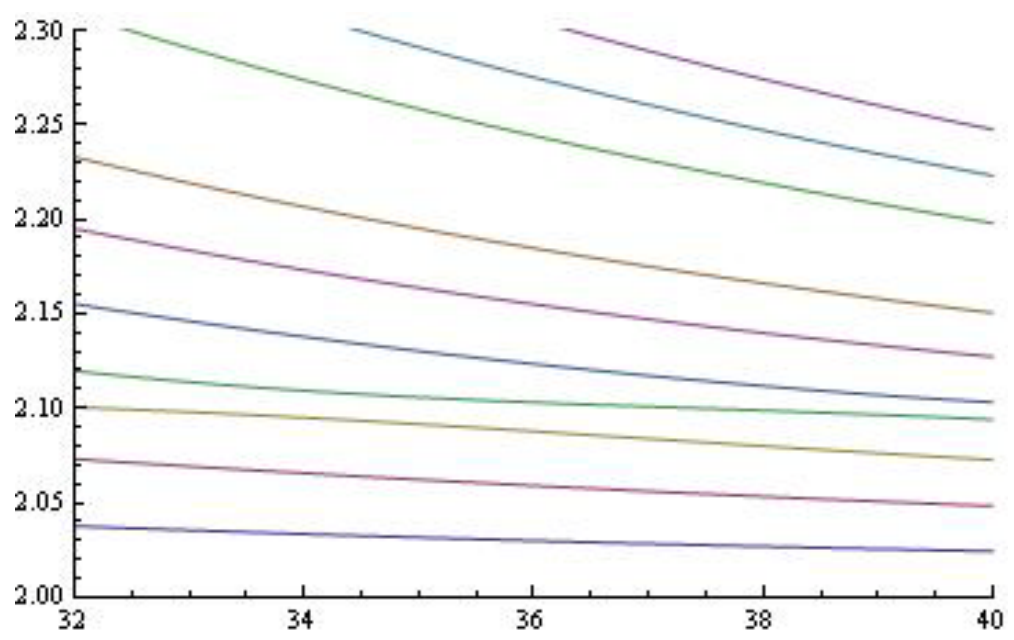

Fig. 4. Energy spectrum with interaction exhibiting avoided level crossing. x-axis: $L\left(1 / m_{\pi}\right)$. y-axis: resonant energy $\left(m_{\pi}\right)$.

We can extract the phase shifts of the states with eigenvalues of the full Hamiltonian at a certain lattice size by using Lüscher's formula.

Lüscher's formula relates the phase shifts of infinite volume scattering in the real world with the energy spectrum, the momentum $k$, and the lattice size $L^{3}$ of the finite volume model. For the case where $l=0$, by Lüscher's formula,

$$
e^{2 i \delta(k)}=\frac{m_{00}+i}{m_{00}-i}=e^{-2 i \phi(q)}
$$




$$
m_{00}=\frac{1}{\pi^{3 / 2} q} Z_{00}\left(1, q^{2}\right)
$$

where $Z_{00}\left(1, q^{2}\right)$ is a generalized zeta function. From Eq.(1) we find:

$$
r \pi-\delta(k)=\phi(q) \text { where } r \in \mathbf{Z},
$$

so that

$$
\delta(k)=r \pi+\arctan \left(\frac{\pi^{3 / 2} q}{Z_{00}\left(1, q^{2}\right)}\right) .
$$

If there is a state $a$ with energy eigenvalue $W_{a}$, then

$$
q_{a}=\frac{L}{2 \pi} \sqrt{2 \mu\left(W_{a}-2 m_{\pi}\right)}
$$

In this case, from (2), we find

$$
\delta\left(k_{a}\right)=r \pi+\arctan \left(\frac{\pi^{3 / 2} q_{a}}{Z_{00}\left(1, q^{2}\right)}\right) .
$$

In this way we can get the phase shift at energy $W_{a}$. We can thus get a track of discretized phase shifts from the energy eigenvalues of the full Hamiltonian at a certain $L$.

The phase shifts of the infinite volume scattering can be calculated by evaluating the $\mathrm{r}$ matrix by

$$
\frac{g^{2}(k)}{2 m_{\pi}+\frac{k^{2}}{2 \mu}-m_{\sigma 0}-2 \mu P \int_{0}^{\infty} \frac{d k^{\prime} k^{\prime 2} g^{2}\left(k^{\prime}\right)}{k^{2}-k^{\prime 2}+i \eta}}=r(E)=-\frac{\tan \delta}{\pi \mu k}
$$

where $P \int_{0}^{\infty} \frac{d k^{\prime} k^{\prime 2} g^{2}\left(k^{\prime}\right)}{k^{2}-k^{\prime 2}+i \eta}$ is the principal value of the integral. Hence we can calculate the infinite volume phase shifts of the two meson scattering with $\sigma$ as the intermediate state. We can compare the phase shifts of the finite volume scattering acquired from Lüscher's formula with the infinite volume phase shifts acquired calculated from the r-matrix. The parameters are chosen so that the resonance position is at 2.1 (with all energies expressed in units of the pion mass). 
A. C. -P. Hsu

\section{Result}

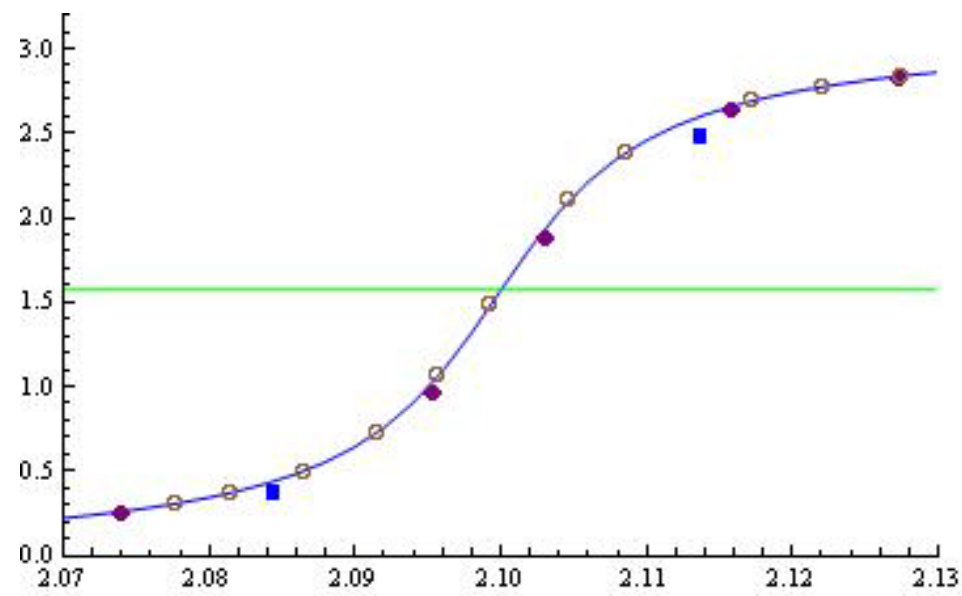

Fig. 5. Phase shift at $\mathrm{L}=20\left(1 / \mathrm{m}_{\pi}\right)$ (squares), $56\left(1 / \mathrm{m}_{\pi}\right)$ (dots), and $90\left(1 / \mathrm{m}_{\pi}\right)$ (circles) compared with the infinite volume phase shifts. $x$-axis: resonant energy $\left(\mathrm{m}_{\pi}\right)$. $\mathrm{y}$-axis: phase shift (rad).

We plot the phase shifts of the finite volume scattering at several lattice sizes together with the infinite volume phase shifts as Fig. 5. We can obtain the resonance position of the finite volume model by Breit-Wigner interpolation, which is shown in Fig. 6.

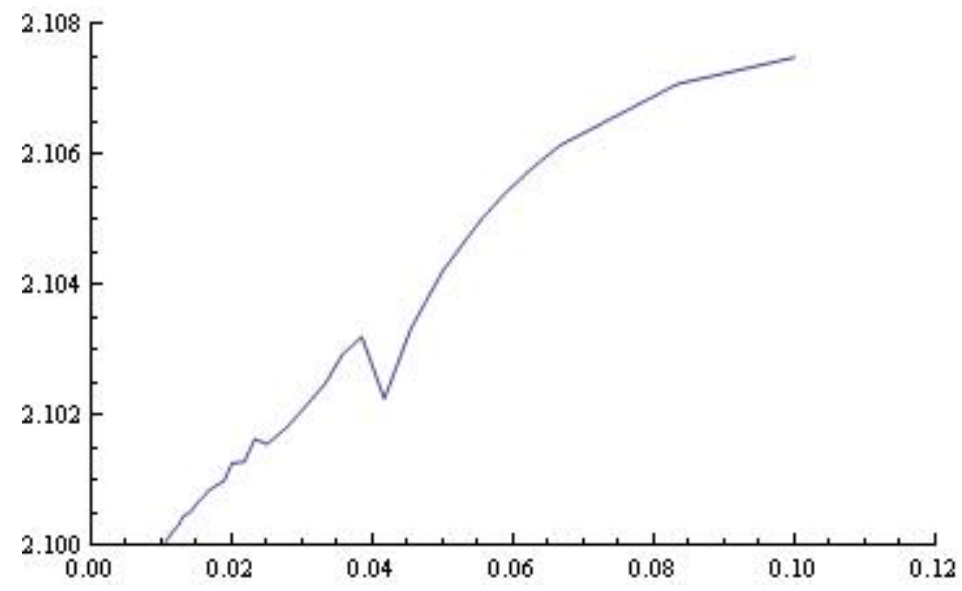

Fig. 6. Resonance position of the finite volume model calculated by Breit-Wegner Interpolation showing its relation with the lattice size. x-axis: $1 / L\left(m_{\pi}\right)$. y-aixs: resonance position $\left(m_{\pi}\right)$.

We find that the larger the lattice size the closer the finite volume phase shift tracks to the infinite volume phase shift curve. Thus, as expected, we find that Lüscher's formula works more precisely in larger lattices. On the other hand, we observe that the deviation from the exact resonance position behaves like $1 / \mathrm{L}$, in contrast with the anticipated exponential suppression of finite volume effects. 
A. C. -P. Hsu

\section{Outlook}

Up to this point we have been dealing with scalar mesons in a non-relativistic setting. In the future, we plan to extend our study to more realistic situations such as the scattering of two pions with $\rho$ as the intermediate state in a relativistic setting. We will apply the model which we have used here to the future studies.

Acknowledgements: This work was supported by the Australian Research Council through an Australian Laureate Fellowship (AWT), a Future Feloowship RDY) and DP110101265 (RDY).

\section{References}

[1] M. Lüscher, Two Particle States on a Torus and Their Relation to the Scattering Matrix, Nucl. Phys. B354 (1991) 531

[2] M. Lüscher , Signatures of Unstable Particles in Finite Volume, Nucl. Phys. B364 (1991) 237 